\title{
Improvement of Traditional Model of Infectious Diseases
}

\author{
Lingzhi Fu \\ School of North China Electric Power University, Baoding 071000, China; \\ 1039716813@qq.com
}

Keywords: epidemic model,multi-objective optimization,Runge-Kutta method

\begin{abstract}
This paper is to speculate the drug demand of each region and assist to plan a reasonable number through analyzing variations of weekly infected people, which is based on epidemic model and multi-objective optimization theory.

Unlike traditional SIR model,I consider the patients under incubation period and antibody carriers after cure in my model. To begin with, I apply five classifications to sample individuals (i.e. the susceptible, borderline cases, confirmed cases, death cases and the cured cases), by which I establish a first order differential system to represent the disease propagation. By applying Runge-Kutta method to establish system, I obtain a fitted curve, through which the future propagation is more predictable.And then,To establish a comprehensive and efficient evaluation model,that is based on previous one while considering other key factors ,including drug manufacturing rate and delivery efficiency, I introduce a comprehensive evaluation coefficient of region coverage intensity .
\end{abstract}

\section{Introduction}

The severe Ebola virus disease(EVD) [1],also known as hemorrhagic fever,its case fatality rate is around $50 \%$. Case fatality rates have varied from $25 \%$ to $90 \%$ in past outbreaks.Ebola spreads through human-to-human transmission via direct contact with the blood, secretions,organs or other bodily fluids of infected people.The incubation period is 2 to 21 days.And what should be taken into concern is that humans are not infectious until they develop symptoms[2].

In order to take a better control of the propagation of EVD,I first carry on the research of the Ebola virus infections model, The difference between my model and common infectious disease model is that ,I based on the core assumptions which are the fixed number population,a number of people could obtain antibody and divided people into groups of susceptible population,already infected population,deceased population,healed population which can be divided into people with antibodies and people without, and some infected people who are still in the incubation period which are asymptomatic and non-infectious.According to the number of population I establish the first order equations and solve them by Runge-Kutta method.I will begin to integrate WHO weekly epidemic data from march,22nd,acquiring the accumulated diagnosed numbers and deaths.Then combine and analysis the overview of the epidemic and the data from the most infected countries based on the model.So as to get a rough transmissions trend.

Based on the model above,I assume bringing the medical control intervebtions and also bringing in evaluation parameters,considering the influence of drugs and vaccine on various people so as to get the ideal trend curve of the Ebola transmission after medical control,and provide a general reference and ideal standards for the model optimization according to terms.Extracting the weekly contrast curve of the number of infections and deaths. In theory each infected person should get treatment,which resulted in the establishment of model,to obtain the weekly demand as far as possible,this ideal drug production rate is an important reference index. 


\section{Definitions:}

Table 1. Model parameters.

\begin{tabular}{ll}
\hline Symbol & Meaning \\
\hline$\lambda$ & Effective contact rate in one week \\
$\alpha$ & Mortality rate in one week \\
$\mu$ & Recovery rate in one week \\
$\beta$ & Rate of rehabilitate with antibodies \\
$\delta$ & Production rate of Suspected cases in one week \\
$\varepsilon$ & Diagnosis rate of suspected cases in one week \\
$p_{1}$ & Probability of new drugs are working \\
$p_{2}$ & Probability of vaccine make susceptible people have antibody \\
$N$ & The total population in Epidemic area \\
$\xi$ & The factor of effective medical coverage \\
$R$ & The maximum coverage radius of medical center \\
\hline
\end{tabular}

\section{Model establishment}

The final purpose lies in considering some critical factors as part of the model to optimize the eradication of Ebola, or at least its current strain. Before this, I need to according to my collected data from March 25,2014 to February 4,2015,to establish model.March 25,2014 for the first day of my model, the Ebola virus propagation model [3].

Inspired by classical infectious disease model, in view of the Ebola virus spread, the population is divided into five types:

The total number of the susceptible group is described by $S(t)$;

The total number of the infected group is described by $I(t)$;

The total number of the recovered group is described by $R(t)$. Among them, according to whether with antibodies can be divided into two categories;

The total number of the suspected group is described by $Y(t)$;

The total number of the deceased group is described by $D(t)$.

For the deceased in incubation period, due to its asymptomatic embodies, and without infectivity, I temporarily to be classified as $\mathrm{S}(\mathrm{t})$.Thus, according to the actual change in the spread of all kinds of people, I roughly drug pharmacokinetics of classical compartment model to describe,As shown in figure 1: 


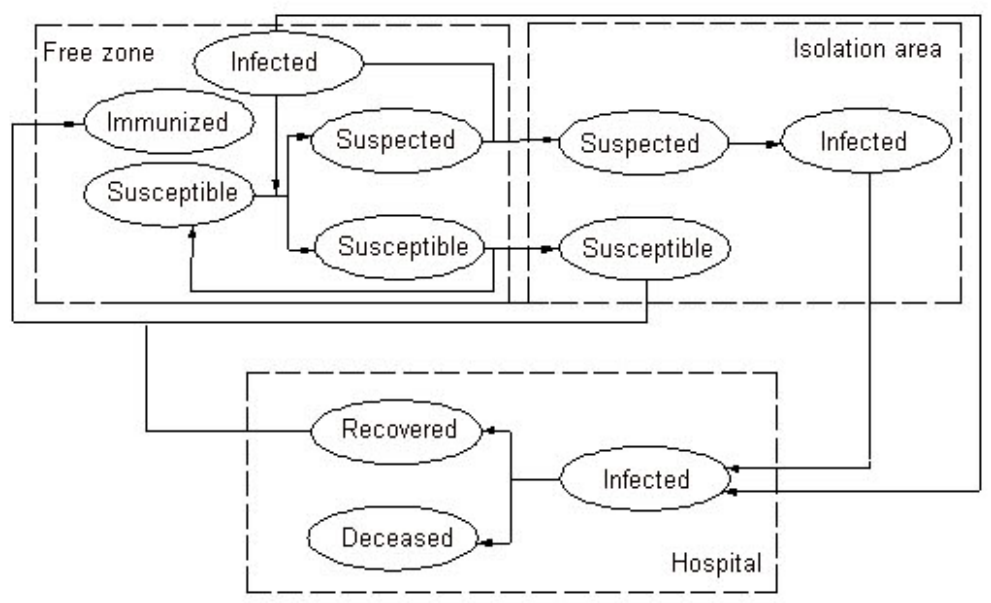

Figure 1.State transition diagram chamber model of the crowd.

A few days ago, The world medical association has announced that their new medication could stop Ebola and cure patients whose disease is not advanced. Thus, I assume that drugs and vaccines from the current come into service, and consider the drug production rate, the condition ,such as drug delivery systems operation situation.Introducing the treatment of the comprehensive evaluation coefficient of effective cover efficiency $\xi$.set up the model.I set up the success rate of the medicine to cure patients is $\mathrm{P}_{1}$, the probability of antibody production after injection of the vaccine is $\mathrm{P}_{2}$,To optimize the virus propagation model, the optimized had an impact on the susceptible, infected people, recovery crowd.I introduce the parameter $\xi$, set up model ,to evaluate the influence of the system model, when take into account the key conditions.When I take medical interventions,respectively consider vaccines and drugs affect the infected and the recovered.thus I get a comprehensive treatment effectiveness evaluation model.And for different values of $\xi$, general analyze the spread of the epidemic and duration[4].

In order to measure the change of the number of people that has antibody, for some of the parameters are optimized model is as follows:

$$
\left\{\begin{array}{l}
\frac{d S(t)}{d t}=-\frac{\lambda}{N} \cdot S(t) \cdot I(t)-\delta \cdot S(t)+(1-\beta) \cdot R(t)-\xi \cdot p_{2} \cdot S(t) \\
\frac{d I(t)}{d t}=\frac{\lambda}{N} \cdot S(t) \cdot I(t)-(\mu+\alpha) \cdot I(t)-\xi \cdot p_{1} I(t) \\
\frac{d R(t)}{d t}=\mu \cdot I(t)-(1-\beta) \cdot R(t)+\xi \cdot p_{1} I(t) \\
\frac{d M(t)}{d t}=\beta \cdot R(t)+\xi \cdot p_{2} \cdot S(t)
\end{array}\right.
$$

\section{Results analysis}

Compared with the virus propagation model,when $\xi=1$, namely all patients to receive treatment.I am fitting out the optimized curve, as shown in figure2. 


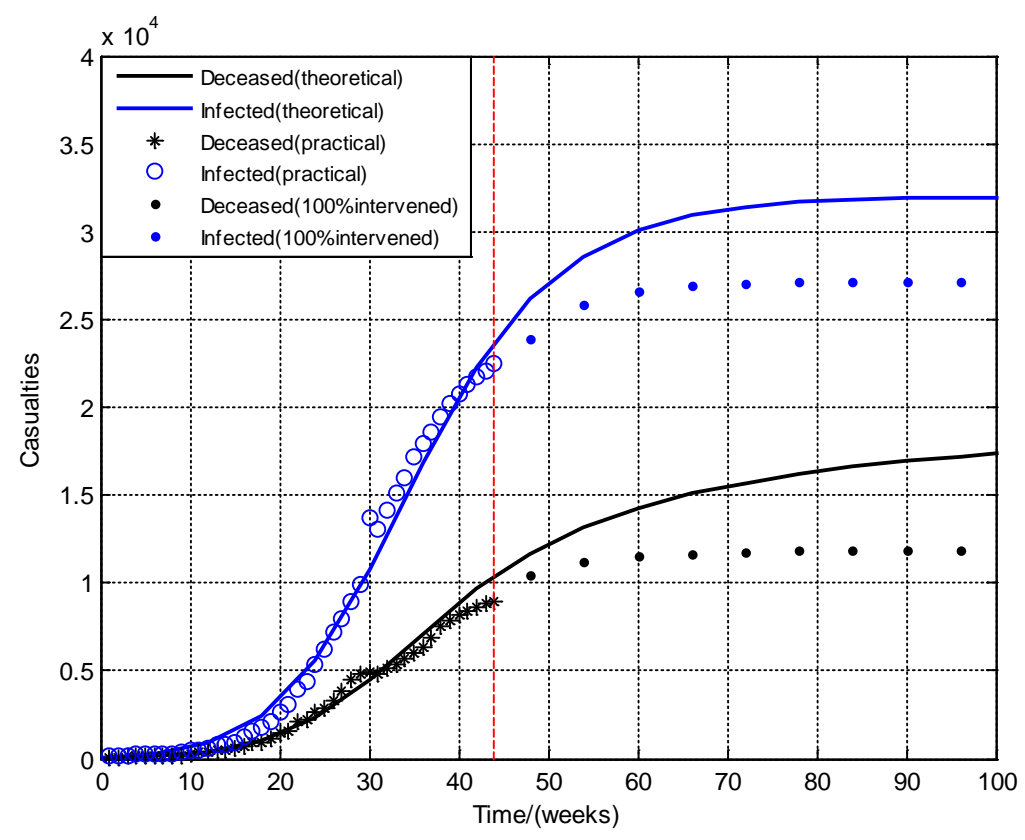

Figure 2. Trends chart of Total.

We can clearly see that after taking medical controls, in the ideal case, the rising trend of the total number of infections and deaths began to slow down. And after 13 weeks, it comes to be zero. According to the provisions of WHO, if there is no new case of infection appears within the period of 6 Ieks which is twice as the Ebola longest incubation period(21 days),the epidemic can be declared as ended[5]. Therefore, I can provide the impact of the key subsequent factors with an relatively ideal reference standard judging by the length of epidemic time.

\section{Summary}

Optimizing the classic SIR model the spread of disease, the population is divided into different types, more detailed consideration, and combined with the effects of a combination of factors of the model, more practical significance.

\section{References}

[1] Zach Yarus ,A Mathematical look at the EbolaVirus.2012.p.1-12.

[2] Gerardo Chowell and Hiroshi Nishiura .Transmission dynamics and control of Ebola virus disease (EVD): a review. Chowell and Nishiura BMC Medicine 2014. p.1-16.

[3] Yifeng Wang,Yi Tian,Qian Yang. Establishment and analysis of mathematical models of SARS.Journal of Engineering Mathematics,2003.p.45-52.

[4] Haiping Zhou,Shaohong Cai.Vulnerable groups sparsely distributed - infection - $\quad$ susceptible disease spread model.Journal of Lanzhou University,2008.p.118-122.

[5] Xiaodong Sun,Haiyin Wang.Emerging infectious diseases prevalent status and control strategies.Shanghai Journal of Preventive Medicine. 